\title{
Arranging numbers on circles to reach maximum total variations
}

\author{
Ying-Jie Liao Min-Zheng Shieh Shi-Chun Tsai \\ Department of Computer Science \\ National Chiao Tung University, Hsinchu 30050, Taiwan \\ $\{$ yjliao,mzhsieh,sctsai\}@csie.nctu.edu.tw
}

Submitted: Jan 15, 2007; Accepted: Jun 10, 2007; Published: Jun 28, 2007

Mathematics Subject Classification: 05A05, 05B30

\begin{abstract}
The dartboard problem is to arrange $n$ numbers on a circle to obtain maximum risk, which is the sum of the $q$-th power of the absolute differences of adjacent numbers, for $q \geq 1$. Curtis showed that the dartboard problem admits a greedy algorithm. We generalize the dartboard problem by considering more circles and the goal is to arrange $k n$ number on $k$ circles to obtain the maximum risk. In this paper, we characterize an optimal arrangement for $k=2$ and show that the generalized dartboard problem also admits a greedy algorithm.
\end{abstract}

\section{Introduction}

Darts is a very popular game. Players throw darts and score points corresponding to the sector the darts just landed on. The traditional dartboard is circular and partitioned into several sectors as shown in figure 1. When playing darts, players often aim at the high score sectors. But for ordinary players, it is hard to land the dart on the desired sectors. The risk of aiming at an area can be measured by the difference between the scores of adjacent sectors. As the larger the difference is, the higher the risk is and the game becomes more challenging. The total risk of a dartboard is the sum over the risks of all sectors. The so called dartboard problem, as discussed in Curtis' paper [4], is to find a cyclic permutation $\tau=\alpha_{1} \cdots \alpha_{n}$ of a multiset $\left\{a_{1}, \cdots, a_{n}\right\}$ on a circle which maximizes the risk function $\sum_{i=1}^{n}\left|\alpha_{i}-\alpha_{i-1}\right|^{q}$ where $\alpha_{0} \equiv \alpha_{n}$ and $q \geq 1$.

The dartboard problem has been studied for a while. Eiselt and Laporte [5] used a branch-and-bound algorithm[1] to find optimal permutations for the dartboard problem on $\{1,2, \ldots, 20\}$ for $q=1$ and $q=2$, and they observed that the traditional dartboard score arrangement is not optimal. Chao and Liang [2] studied the permutations of $n$ distinct numbers arranged on a circle or a line and showed the arrangements that maximize or 


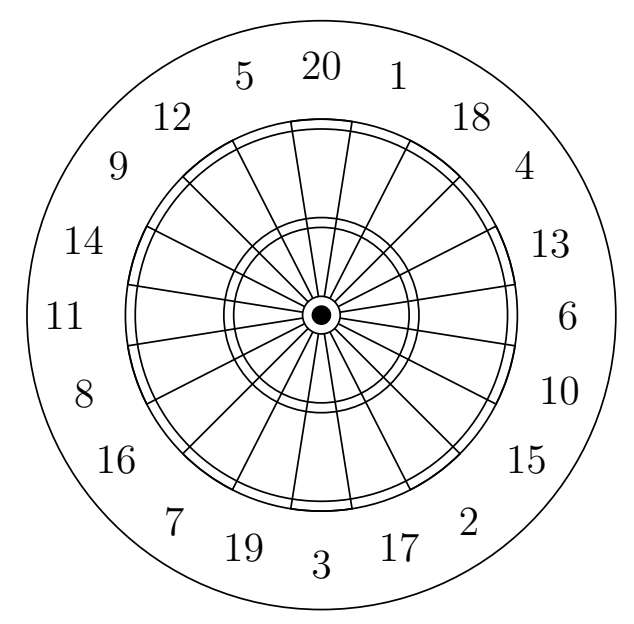

Figure 1: A traditional dartboard.

minimize the risk function. Later, Cohen and Tonkes [3] analyzed optimal permutations for multisets of numbers. Recently, Curtis[4] designed a greedy algorithm to find an optimal permutation $\pi=a_{1} a_{n-1} a_{3} a_{n-3} a_{5} \cdots a_{n-4} a_{4} a_{n-2} a_{2} a_{n}$ for the dartboard problem, where $a_{1} \leq a_{2} \leq \cdots \leq a_{n}$.

In this paper, we extend the dartboard problem from single circle to double circles. For example, the dartboard with two circles, is as shown in figure 2. Assume that we are given a multiset of $2 n$ numbers and a double layer dartboard. We use a pair of permutations $\left(v_{1} \cdots v_{n}, w_{1} \cdots w_{n}\right)$ to describe the arrangement, as shown in figure 3 , where $v_{1} \cdots v_{n}$ is a cyclic permutation for the outer circle and $w_{1} \cdots w_{n}$ is a cyclic permutation for the inner circle. We can extend the definition of the risk function to the double layer dartboard. For example, the risk of the arrangement $\left(v_{1} \cdots v_{n}, w_{1} \cdots w_{n}\right)$ in figure 3 , denoted by $r_{q}\left(v_{1} \cdots v_{n}, w_{1} \cdots w_{n}\right)$, is defined as $\sum_{i=1}^{n}\left|v_{i}-w_{i}\right|^{q}+\sum_{i=1}^{n}\left|v_{i}-v_{i-1}\right|^{q}+$ $\sum_{i=1}^{n}\left|w_{i}-w_{i-1}\right|^{q}$ where $v_{0} \equiv v_{n}$ and $w_{0} \equiv w_{n}$. We define the 2-dartboard problem as: finding an arrangement $\left(\tau_{V}, \tau_{W}\right)$ for a multiset $A=\left\{a_{1}, \cdots, a_{2 n}\right\}$ on two circles which maximizes the risk function, where $V$ and $W$ is a partition of $A$ and both have $n$ elements.

Furthermore, we can extend the dartboard problem to $k$-layer dartboard. We use $k$ cyclic permutations $\left(\tau_{1}, \cdots, \tau_{k}\right)$ to represents the arrangement where $\tau_{i}$ is a permutation on $n$ elements for the $i$-th circle. The risk function can be recursively defined as

$r_{q}\left(\tau_{1}, \cdots, \tau_{k-2}, \tau_{k-1}=v_{1} \cdots v_{n}, \tau_{k}=w_{1} \cdots w_{n}\right)=r_{q}\left(\tau_{1}, \cdots, \tau_{k-1}\right)+r_{q}\left(\tau_{k}\right)+\sum_{i=1}^{n}\left|v_{i}-w_{i}\right|^{q}$,

where the last term is the sum over the $q$-th power of the absolute differences between numbers of the $(k-1)$-th and $k$-th circles. Similarly, the $k$-dartboard problem is: finding an arrangement for a multiset $A=\left\{a_{1}, \cdots, a_{k n}\right\}$ on $k$ circles to maximize the risk function.

For the $k$-dartboard problem, we show once the numbers on each circle is determined, then we can find the maximum arrangement efficiently. Moreover, we show that for the 2-dartboard problem, there exists an efficient greedy algorithm given an arbitrary input. 


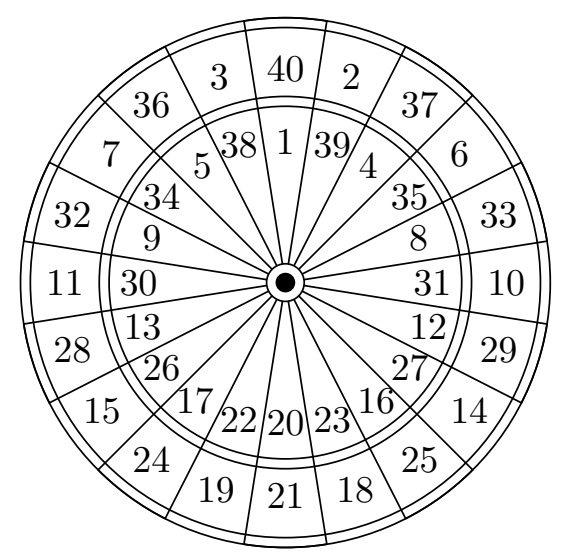

Figure 2: A double layer dartboard

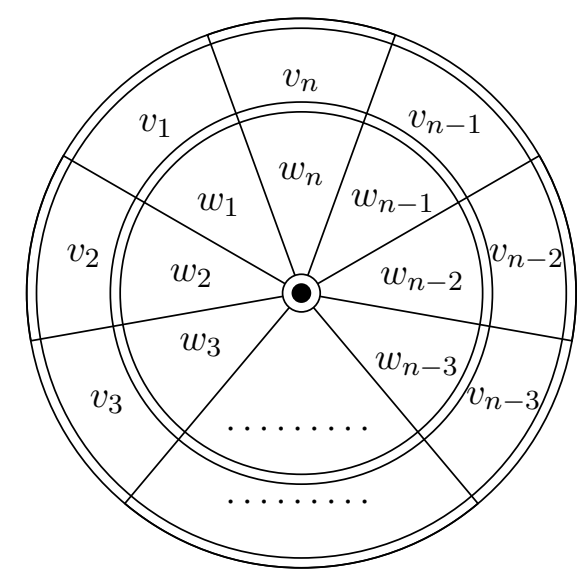

Figure 3: An arrangement for double layer dartboard

However, it is not clear whether there exist an efficient algorithm for the k-dartboard problem $(k>2)$ when the input does not specify the numbers on each circle. We leave it as an open question.

\section{Preliminaries}

The following lemma is very useful in our proof, which was proved in Curtis' paper[4].

Lemma 1. [4] Let $l_{\text {min }}, l_{\text {max }}, r_{\min }, r_{\max }, q$ be real numbers with $q \geq 1$. If $l_{\min } \leq l_{\max }$ and $r_{\text {min }} \leq r_{\text {max }}$, then $\left|l_{\text {max }}-r_{\text {min }}\right|^{q}+\left|l_{\text {min }}-r_{\text {max }}\right|^{q} \geq\left|l_{\text {max }}-r_{\max }\right|^{q}+\left|l_{\text {min }}-r_{\text {min }}\right|^{q}$.

With lemma 1, Curtis[4] proved the following theorem:

Theorem 1. [4] For arranging n numbers $a_{1} \leq a_{2} \leq \cdots \leq a_{n}$ on a single circle dartboard, the permutation $a_{1} a_{n-1} a_{3} a_{n-3} a_{5} \cdots a_{n-4} a_{4} a_{n-2} a_{2} a_{n}$ maximizes the risk function. 
For an $n$-element multiset $A$, we denote the maximum permutation of $A$ claimed in Theorem 1 by $\pi_{n}(A)$. Cyclic permutations are reverse-invariant and shift-invariant when calculating the risk function. That is, the value of risk is the same under the following permutations $\alpha_{1} \cdots \alpha_{n}, \alpha_{n} \cdots \alpha_{1}$ and $\alpha_{i+1} \cdots \alpha_{n} \alpha_{1} \cdots \alpha_{i}$ for $i \in[n-1]$. We denote the reverse of permutation $\tau$ by $\tau^{R}$.

Lemma 2. Given two multisets of numbers $X=\left\{x_{1}, \cdots, x_{n}\right\}$ and $Y=\left\{y_{1}, \cdots, y_{n}\right\}$. Assume that $x_{1} \leq \cdots \leq x_{n}$. If $y_{1} \leq \cdots \leq y_{n}$, then $\sum_{i=1}^{n}\left|x_{i}-y_{n-i+1}\right|^{q}$ has the maximum value over all possible permutations of $y_{i}$ 's, where $q \geq 1$.

Proof. Assume that $y_{1}, \cdots, y_{n}$ are not sorted in increasing order and $\sum_{i=1}^{n}\left|x_{i}-y_{n-i+1}\right|^{q}$ is maximized. Thus, there exists $i, j$ such that $i<j$ and $y_{n-i+1}<y_{n-j+1}$. We call $i$ and $j$ form an inversion in $y_{i}$ 's. As $x_{i} \leq x_{j}$, we know that $\left|x_{i}-y_{n-i+1}\right|^{q}+\left|x_{j}-y_{n-j+1}\right|^{q} \leq$ $\left|x_{j}-y_{n-i+1}\right|^{q}+\left|x_{i}-y_{n-j+1}\right|^{q}$ by lemma 1. Therefore the sum does not decrease after swapping $y_{n-i+1}$ and $y_{n-j+1}$. By repeating the swapping step whenever there is an inversion in $y_{i}$ 's, then we can eventually rearrange $y_{i}$ 's in increasing order without decreasing the sum, since there are at most $O\left(n^{2}\right)$ inversions in a permutation of size $n$.

With lemma 2, we have the following theorem.

Theorem 2. If $n$ numbers on each circle are given, say $X$ and $Y$ are the multisets of numbers on the outer circle and the inner circle, respectively, then the arrangement $\left(\pi_{n}(X), \pi_{n}(Y)^{R}\right)$, achieves the maximum risk. That is, $r_{q}\left(\pi_{n}(X), \pi_{n}(Y)^{R}\right) \geq r_{q}\left(\tau_{X}, \tau_{Y}\right)$ for any permutation $\tau_{X}$ of $X$ and $\tau_{Y}$ of $Y$.

Proof. Since the numbers on the outer circle are permuted with $\pi_{n}(X)$, the risk contributed from the outer circle is maximized and so is $\pi_{n}(Y)^{R}$ to the inner circle. Assume $X=\left\{x_{1}, \cdots, x_{n}\right\}$ with $x_{1} \leq \cdots \leq x_{n}$ and $Y=\left\{y_{1}, \cdots, y_{n}\right\}$ with $y_{1} \leq \cdots \leq y_{n}$. Observe that $\pi_{n}(X)=x_{1} x_{n-1} x_{3} \cdots x_{n-2} x_{2} x_{n}$ and $\pi_{n}(Y)^{R}=y_{n} y_{2} y_{n-2} \cdots y_{3} y_{n-1} y_{1}$. By lemma 2, we have the risk contributed from the difference between circles is maximized since $x_{i}$ is adjacent to $y_{n-i+1}$. Therefore, we conclude that $r_{q}\left(\pi_{n}(X), \pi_{n}(Y)^{R}\right) \geq r_{q}\left(\tau_{X}, \tau_{Y}\right)$ for every permutation $\tau_{X}$ of $X$ and $\tau_{Y}$ of $Y$.

By the above, for convenience, we denote the maximum risk corresponding to partition $(X, Y)$ by $r_{q}(X, Y)$.

Corollary 1. Let $X_{i}$ be the multiset of $n$ numbers on the $i$-th circle, $i=1 . . k$, then the arrangement, permuting circle $i$ with $\pi_{n}\left(X_{i}\right)$ if $i$ is odd, else with $\pi_{n}\left(X_{i}\right)^{R}$, achieves the maximum risk.

Proof. By induction on $k$, assume the corollary is true up to $k-1$. Similar to the proof for theorem 2, the risks contributed from the first $k-1$ circles and from the $k$-th circle are maximized by induction basis. The risk contributed from the difference between the $(k-1)$-th and $k$-th circles is also maximized due to lemma 2 . Thus the corollary is true for $k$.

Let $\mathrm{A}$ be a multiset of $k n$ elements and $\left(A_{1}, \cdots, A_{k}\right)$ is a partition of $A$ with each $A_{i}$ of the same size. We say a partition $\left(A_{1}, \cdots, A_{k}\right)$ is maximum if $r_{q}\left(\pi_{n}\left(A_{1}\right), \pi_{n}\left(A_{2}\right)^{R}, \cdots\right)$ $\geq r_{q}\left(\tau_{1}, \cdots, \tau_{k}\right)$, for every arrangement $\left(\tau_{1}, \cdots, \tau_{k}\right)$ of $A$. Note that corollary 1 implies 
Algorithm GreedyPartition $\left(\left\{a_{1}, \cdots, a_{2 n}\right\}\right)$

1. if $n=3$ then return $\left(\left\{a_{1}, a_{2 n-2}, a_{2 n-1}\right\},\left\{a_{2}, a_{3}, a_{2 n}\right\}\right)$

2. if $n=4$ then return $\left(\left\{a_{1}, a_{4}, a_{2 n-2}, a_{2 n-1}\right\},\left\{a_{2}, a_{3}, a_{2 n-3}, a_{2 n}\right\}\right)$

3. $\left(X^{\prime}, Y^{\prime}\right)=\operatorname{GreEdyPARTITION}\left(\left\{a_{3}, \cdots, a_{2 n-2}\right\}\right)$;

4. $X \leftarrow Y^{\prime} \cup\left\{a_{1}, a_{2 n-1}\right\}, Y \leftarrow X^{\prime} \cup\left\{a_{2 n}, a_{2}\right\}$;

5. $\operatorname{return}(X, Y)$;

Figure 4: Our greedy algorithm

that once the partition $\left(A_{1}, \cdots, A_{k}\right)$ of $k n$ numbers is determined, the maximum possible risk achieved by $\left(A_{1}, \cdots, A_{k}\right)$ can be determined, so we can just focus on finding a partition that yields the maximum risk.

\section{Optimal arrangement for 2-dartboard problem}

In this section, we show how to solve the 2-dartboard problem with a greedy method. Consider a multiset $\left\{a_{1}, \cdots, a_{2 n}\right\}$ with $a_{1} \leq \cdots \leq a_{2 n}$. By theorem 2 , we focus on finding a maximum partition. But trying all possible $\left(\begin{array}{c}2 n \\ n\end{array}\right)$ partitions is inefficient. Here we propose an efficient greedy method to obtain a maximum partition, as in figure 4 .

Theorem 3. There is an efficient algorithm solving the 2-dartboard problem.

Proof. There are only $\left(\begin{array}{l}2 \\ 1\end{array}\right)=2$ and $\left(\begin{array}{l}4 \\ 2\end{array}\right)=6$ possible partitions when $n=1$ and $n=2$, respectively, so we can find out the maximum partition efficiently by brute force if $n \leq 2$. When $n \geq 3$, we claim that GREEDYPARTITION algorithm gives a maximum partition. The correctness of a greedy algorithm can be justified by checking the greedy choice property and the property of optimal substructure. To prove the greedy choice property of GreEdyPARTition, we need to show that there exists a maximum partition $(X, Y)$ with $\left\{a_{1}, a_{2 n-1}\right\} \subseteq X$ and $\left\{a_{2}, a_{2 n}\right\} \subseteq Y$. To prove the optimal substructure property, we need to show that there exists a maximum partition $(X, Y)$ such that $\left(Y-\left\{a_{2}, a_{2 n}\right\}, X-\right.$ $\left.\left\{a_{1}, a_{2 n-1}\right\}\right)$ is also a maximum partition for the subproblem with instance $\left\{a_{3}, \cdots, a_{2 n-2}\right\}$. The proof of correctness consists of 4 propositions. The greedy choice property is proved by proposition 1 and 2 and the optimal substructure is proved by proposition 3 and 4 .

Proposition 1. For $n \geq 3$, there exists a maximum partition $\left(X^{*}, Y^{*}\right)$ such that $a_{1} \in X^{*}$ and $a_{2 n} \in Y^{*}$.

Proof. Let $(X, Y)$ be another maximum partition. Let $X=\left\{x_{1}, \cdots, x_{n}\right\}$ with $x_{1} \leq \cdots \leq$ $x_{n}$ and $Y=\left\{y_{1}, \cdots, y_{n}\right\}$ with $y_{1} \leq \cdots \leq y_{n}$. By theorem $2,\left(x_{1} x_{n-1} x_{3} \cdots x_{n-2} x_{2} x_{n}, y_{n} y_{2}\right.$ $\left.y_{n-2} \cdots y_{3} y_{n-1} y_{1}\right)$ is an optimal arrangement. Without loss of generality, we can assume $x_{1}=a_{1}$. Note that $a_{2 n}$ can be either $y_{n}$ or $x_{n}$. If $y_{n}=a_{2 n}$, then we're done. Thus we assume $x_{n}=a_{2 n}$.

Since $x_{1}=a_{1}$ and $x_{n}=a_{2 n}$, we have $x_{1} \leq y_{1}$ and $x_{n} \geq y_{n}$. Note if $x_{1}=y_{1}$ or $x_{n}=y_{n}$, then $(Y, X)$ satisfies the proposition. Hence, we consider $x_{1}<y_{1}$ and $x_{n}>y_{n}$ from now 
on. Let $l=\min \left\{i: x_{i} \geq y_{i}\right\}$ and $r=\min \left\{j: x_{n-j+1} \leq y_{n-j+1}\right\}$. Let $k=\min (l, r)$. It is clear that $1<k<n$, and for every $i<k, x_{i}<y_{i}$ and $x_{n-i+1}>y_{n-i+1}$. By lemma 1, we have

$$
\left|x_{i}-y_{n-i+1}\right|^{q}+\left|x_{n-i+1}-y_{i}\right|^{q} \leq\left|x_{i}-x_{n-i+1}\right|^{q}+\left|y_{i}-y_{n-i+1}\right|^{q}
$$

for every $i<k$. Thus, swapping $x_{i}$ 's with $y_{i}$ 's and swapping $x_{n-i+1}$ 's with $y_{n-i+1}$ 's respectively, for every $i<k$ will not decrease the risk contributed from the difference between circles. This kind of swapping is a basic step of our argument. The rest part of proof is to decide the numbers we should swap. There are two possible cases:

- $k=l<r$ : For $k$ is odd, we swap $x_{n-k+2}, x_{k-2}, x_{n-k+4}, x_{k-4}, \cdots, x_{n-1}, a_{1}$ with $y_{n-k+2}, y_{k-2}, y_{n-k+4}, y_{k-4}, \cdots, y_{n-1}, y_{1}$, respectively. We illustrate the swapping operation in figure 5 . For $k$ is even, as in figure 6 , we swap $x_{n-k+2}, x_{k-2}, x_{n-k+4}$, $x_{k-4}, \cdots, x_{2}, a_{2 n}$ with $y_{n-k+2}, y_{k-2}, y_{n-k+4}, y_{k-4}, \cdots, y_{2}, y_{n}$, respectively.
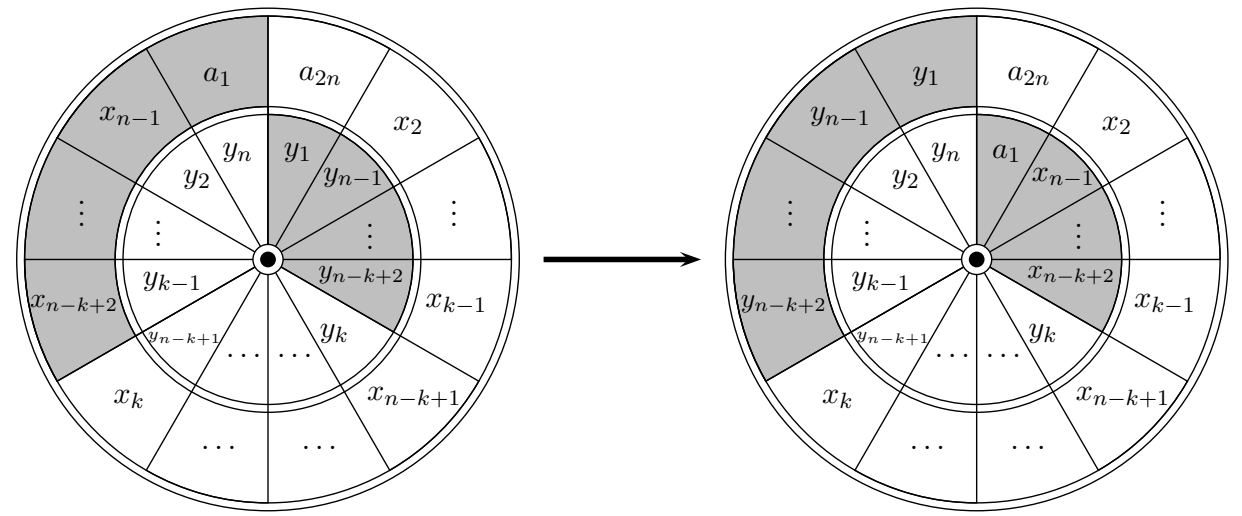

Figure 5: The swapping operation when $k=l$ and $k$ is odd
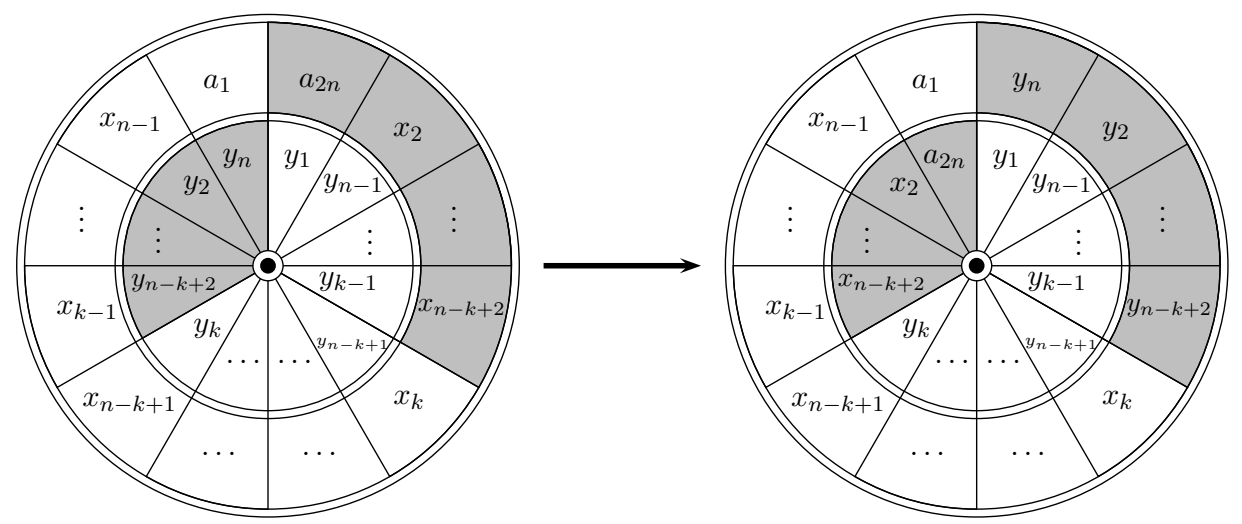

Figure 6: The swapping operation when $k=l$ and $k$ is even

The swapping operation exchanges the elements in the gray regions. The new arrangement has $a_{1}$ and $a_{2 n}$ on different circles. As mentioned above, swapping the numbers in the gray regions does not decrease the risk from the difference between circles. Moreover, the illustrations indicate that the neighbors of $a_{1}, a_{2 n}, y_{1}$ and $y_{n}$ 
are not changed. Hence the only possibility that swapping may decrease the risk is from the two pairs $\left(x_{k}, x_{n-k+2}\right)$ and $\left(y_{k}, y_{n-k+2}\right)$ which may have higher risk sum than $\left(x_{k}, y_{n-k+2}\right)$ and $\left(y_{k}, x_{n-k+2}\right)$ do. However, since $k=l$, we have $x_{k} \geq y_{k}$ and $x_{n-k+2}>y_{n-k+2}$. By lemma 1, we have

$$
\left|x_{k}-x_{n-k+2}\right|^{q}+\left|y_{k}-y_{n-k+2}\right|^{q} \leq\left|x_{k}-y_{n-k+2}\right|^{q}+\left|y_{k}-x_{n-k+2}\right|^{q} .
$$

Thus the risk function does not decrease after the swapping operation.

- $k=r \leq l$ : For $k$ is odd, we swap $x_{k-1}, x_{n-k+3}, x_{k-3}, x_{n-k+5} \cdots, x_{2}, a_{2 n}$ with $y_{k-1}, y_{n-k+3}, y_{k-3}, y_{n-k+5}, \cdots, y_{2}, y_{n}$, respectively, as in figure 7 . For $k$ is even, we swap $x_{k-1}, x_{n-k+3}, x_{k-3}, x_{n-k+5} \cdots, x_{n-1}, a_{1}$ with $y_{k-1}, y_{n-k+3}, y_{k-3}, y_{n-k+5}, \cdots, y_{n-1}$, $y_{1}$, respectively, as in figure 8 .

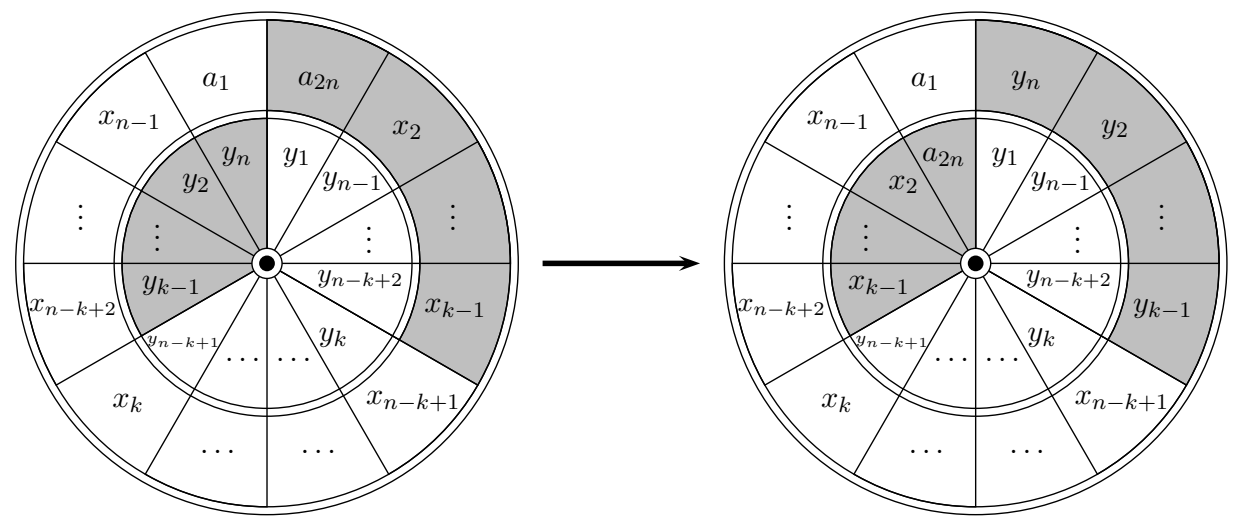

Figure 7: The swapping operation when $k=r$ and $k$ is odd
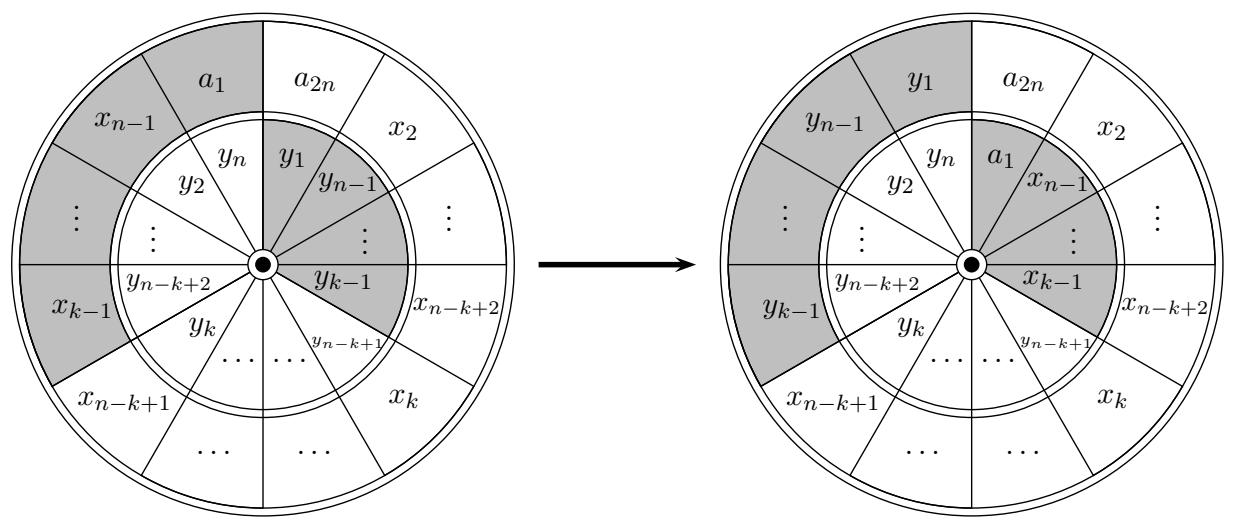

Figure 8: The swapping operation when $k=r$ and $k$ is even

Similarly, the swapping operation puts $a_{1}$ and $a_{2 n}$ on different circles, and the only possibility that swapping may decrease the risk is from the two pairs $\left(x_{n-k+1}, x_{k-1}\right)$ and $\left(y_{n-k+1}, y_{k-1}\right)$ which may have higher risk sum than $\left(x_{n-k+1}, y_{k-1}\right)$ and $\left(y_{n-k+1}\right.$, $\left.x_{k-1}\right)$ do. However, due to $k=r$, we have $x_{n-k+1} \leq y_{n-k+1}$ and $x_{k-1}<y_{k-1}$. By 
lemma 1, we have

$$
\left|x_{k-1}-x_{n-k+1}\right|^{q}+\left|y_{k-1}-y_{n-k+1}\right|^{q} \leq\left|x_{k-1}-y_{n-k+1}\right|^{q}+\left|y_{k-1}-x_{n-k+1}\right|^{q}
$$

Again, swapping does not decrease the risk function.

We conclude that there exists a maximum arrangement in the required form.

Proposition 2. For $n \geq 3$, there exists a maximum partition $\left(X^{*}, Y^{*}\right)$ such that $a_{1}, a_{2 n-1}$ $\in X^{*}$ and $a_{2}, a_{2 n} \in Y^{*}$.

Proof. Let $(X, Y)$ be an arbitrary maximum partition. Let $X=\left\{x_{1}, \cdots, x_{n}\right\}$ with $x_{1} \leq$ $\cdots \leq x_{n}$ and $Y=\left\{y_{1}, \cdots, y_{n}\right\}$ with $y_{1} \leq \cdots \leq y_{n}$. By proposition 1 , we can assume $x_{1}=a_{1}$ and $y_{n}=a_{2 n}$. If $a_{2} \notin Y$, then $x_{2}=a_{2}$ since $a_{2}$ is the second smallest element. We obtain another arrangement with $a_{2}$ on the inner circle by swapping $a_{2}$ and $y_{1}$, as in the following illustration:

\begin{tabular}{c|c|c|c|c|c}
\multicolumn{7}{c}{ Before swapping } \\
\hline$\cdots$ & $a_{1}$ & $x_{n}$ & $a_{2}$ & $x_{n-2}$ & $\cdots$ \\
\hline$\cdots$ & $a_{2 n}$ & $y_{1}$ & $y_{n-1}$ & $y_{3}$ & $\cdots$ \\
\hline
\end{tabular}

\begin{tabular}{c|c|c|c|c|c}
\multicolumn{7}{c}{ After swapping } \\
\hline$\cdots$ & $a_{1}$ & $x_{n}$ & $y_{1}$ & $x_{n-2}$ & $\cdots$ \\
\hline$\cdots$ & $a_{2 n}$ & $a_{2}$ & $y_{n-1}$ & $y_{3}$ & $\cdots$ \\
\hline
\end{tabular}

It is clear that $a_{2} \leq y_{1}$ and $x_{n-2} \leq a_{2 n}$. By lemma 1, we have $\left|a_{2 n}-y_{1}\right|^{q}+\left|x_{n-2}-a_{2}\right|^{q} \leq$ $\left|a_{2 n}-a_{2}\right|^{q}+\left|x_{n-2}-y_{1}\right|^{q}$. Therefore the swapping operation does not decrease the risk and the new arrangement is maximum. Hence, we can assume $a_{2} \in Y$ from now on.

If $a_{2 n-1} \notin X$, then $y_{n-1}=a_{2 n-1}$ since $a_{2 n-1}$ is the second largest element. Similarly, we can swap $a_{2 n-1}$ with $x_{n}$ to obtain an arrangement with $a_{2 n-1}$ on the outer circle:

Before swapping

\begin{tabular}{c|c|c|c|c|c}
\hline$\cdots$ & $a_{1}$ & $x_{n}$ & $x_{2}$ & $x_{n-2}$ & $\cdots$ \\
\hline$\cdots$ & $a_{2 n}$ & $a_{2}$ & $a_{2 n-1}$ & $y_{3}$ & $\cdots$ \\
\hline
\end{tabular}

After swapping

\begin{tabular}{c|c|c|c|c|c}
\hline$\cdots$ & $a_{1}$ & $a_{2 n-1}$ & $x_{2}$ & $x_{n-2}$ & $\cdots$ \\
\hline$\cdots$ & $a_{2 n}$ & $a_{2}$ & $x_{n}$ & $y_{3}$ & $\cdots$ \\
\hline
\end{tabular}

It is clear that $a_{2 n-1} \geq x_{n}$ and $y_{3} \geq a_{1}$. By lemma 1, we have $\left|a_{2 n-1}-y_{3}\right|^{q}+\left|x_{n}-a_{1}\right|^{q} \leq$ $\left|a_{2 n-1}-a_{1}\right|^{q}+\left|x_{n}-y_{3}\right|^{q}$. The swapping operation does not decrease the risk. We conclude that there exists a maximum partition satisfying the proposition.

Proposition 3. For $n \geq 3$, there exists a maximum partition $\left(X^{*}, Y^{*}\right)$ such that $a_{1}, a_{2 n-1}$, $a_{2 n-2} \in X^{*}$ and $a_{2}, a_{3}, a_{2 n} \in Y^{*}$.

Proof. Let $(X, Y)$ be a maximum partition. Let $X=\left\{x_{1}, \cdots, x_{n}\right\}$ with $x_{1} \leq \cdots \leq x_{n}$ and $Y=\left\{y_{1}, \cdots, y_{n}\right\}$ with $y_{1} \leq \cdots \leq y_{n}$. By proposition 2 , let $x_{1}=a_{1}, x_{n}=a_{2 n-1}$, $y_{1}=a_{2}$ and $y_{n}=a_{2 n}$. There are 3 disjoint possible cases such that $(X, Y)$ does not satisfy the proposition. We will reduce them to the required form case by case.

- Case 1: " $a_{3} \in X$ and $a_{2 n-2} \in Y$." By theorem 2, we can assume $x_{2}=a_{3}$ and $y_{n-1}=a_{2 n-2}$. Note that this is the only case that $(X, Y)$ does not satisfy the 
proposition when $n=3$. In this case, we can rotate the 2 -by-2 block, which contains $a_{1}, a_{2}, a_{2 n-1}$ and $a_{2 n}, 180$ degrees:

Before rotation

\begin{tabular}{c|c|c|c|c|c}
\hline$\cdots$ & $x_{n-1}$ & $a_{1}$ & $a_{2 n-1}$ & $a_{3}$ & $\cdots$ \\
\hline$\cdots$ & $y_{2}$ & $a_{2 n}$ & $a_{2}$ & $a_{2 n-2}$ & $\cdots$ \\
\hline
\end{tabular}

After rotation

\begin{tabular}{c|c|c|c|c|c}
\hline$\cdots$ & $x_{n-1}$ & $a_{2}$ & $a_{2 n}$ & $a_{3}$ & $\cdots$ \\
\hline$\cdots$ & $y_{2}$ & $a_{2 n-1}$ & $a_{1}$ & $a_{2 n-2}$ & $\cdots$ \\
\hline
\end{tabular}

Since $a_{1} \leq a_{2}$ and $a_{2 n-2} \geq x_{n-1}$, we have

$$
\left|a_{1}-x_{n-1}\right|^{q}+\left|a_{2}-a_{2 n-2}\right|^{q} \leq\left|a_{1}-a_{2 n-2}\right|^{q}+\left|a_{2}-x_{n-1}\right|^{q} .
$$

Similarly, since $a_{2 n} \geq a_{2 n-1}$ and $a_{3} \leq y_{2}$ we have

$$
\left|a_{2 n-1}-a_{3}\right|^{q}+\left|a_{2 n}-y_{2}\right|^{q} \leq\left|a_{2 n}-a_{3}\right|^{q}+\left|a_{2 n-1}-y_{2}\right|^{q} \text {. }
$$

Therefore, the rotation operation does not decrease risk. It yields a maximum partition as required.

- Case 2: " $a_{3} \in X$ and $a_{2 n-2} \in X$." By theorem 2, we have $x_{2}=a_{3}$ and $x_{n-1}=a_{2 n-2}$. Moreover, we can assume that $y_{2}>a_{3}$ and $y_{n-1}<a_{2 n-2}$, since if $y_{2}=a_{3}$ or $y_{n-1}=a_{2 n-2}$ then it reduces to case 1 . Now we can swap $a_{2 n-1}$ with $a_{2 n}$ as follows:

\begin{tabular}{c|c|c|c|c|c}
\multicolumn{7}{c}{ Before swapping } \\
\hline$\cdots$ & $a_{2 n-2}$ & $a_{1}$ & $a_{2 n-1}$ & $a_{3}$ & $\cdots$ \\
\hline$\cdots$ & $y_{2}$ & $a_{2 n}$ & $a_{2}$ & $y_{n-1}$ & $\cdots$ \\
\hline
\end{tabular}

\begin{tabular}{c|c|c|c|c|c}
\multicolumn{7}{c}{ After swapping } \\
\hline$\cdots$ & $a_{2 n-2}$ & $a_{1}$ & $a_{2 n}$ & $a_{3}$ & $\cdots$ \\
\hline$\cdots$ & $y_{2}$ & $a_{2 n-1}$ & $a_{2}$ & $y_{n-1}$ & $\cdots$ \\
\hline
\end{tabular}

Since $a_{2 n-1} \leq a_{2 n}$ and $a_{3}<y_{2}$, we know the swapping operation does not decrease risk. Since $x_{2}=a_{3}<y_{2}$ and $y_{n-1}<a_{2 n-2}=x_{n-1}$, we can apply similar swapping operations as in the proof of proposition 1 with $k>2$. Depending on the values of $k$ and $n$, the adjustment will yield to one of the following arrangements:

Swapping upper-left with lower right

\begin{tabular}{c|c|c|c|c|l}
\hline$\cdots$ & $y_{n-1}$ & $a_{2}$ & $a_{2 n}$ & $a_{3}$ & $\cdots$ \\
\hline$\cdots$ & $y_{2}$ & $a_{2 n-1}$ & $a_{1}$ & $a_{2 n-2}$ & $\cdots$ \\
\hline
\end{tabular}

Swapping upper-right with lower-left

\begin{tabular}{c|c|c|c|c|l}
\hline$\cdots$ & $a_{2 n-2}$ & $a_{1}$ & $a_{2 n-1}$ & $y_{2}$ & $\cdots$ \\
\hline$\cdots$ & $a_{3}$ & $a_{2 n}$ & $a_{2}$ & $y_{n-1}$ & $\cdots$ \\
\hline
\end{tabular}

However, both cases yield a maximum partition as required.

- Case 3: " $a_{3} \in Y$ and $a_{2 n-2} \in Y$." In this case, we swap $a_{1}$ and $a_{2}$, and apply similar operations as in proposition 1 to obtain an arrangement which yields a partition as required. The analysis is analogous to case 2 .

From the above, this completes the proof of this proposition.

Proposition 4. For $n \geq 4$, there exists a maximum partition $\left(X^{*}, Y^{*}\right)$ such that $a_{1}, a_{4}$, $a_{2 n-2}, a_{2 n-1} \in X^{*}$ and $a_{2}, a_{3}, a_{2 n-3}, a_{2 n} \in Y^{*}$. Moreover, for $n>4$, suppose $(X, Y)$ is a maximum partition satisfying proposition 2 for the sub-instance $\left\{a_{3}, \cdots, a_{2 n-2}\right\}$, then $\left(Y \cup\left\{a_{1}, a_{2 n-1}\right\}, X \cup\left\{a_{2}, a_{2 n}\right\}\right)$ is a maximum partition. 
Proof. First, we prove the "moreover" part. Since $a_{1} \leq a_{2} \cdots \leq a_{2 n}$ and $(X, Y)$ satisfies proposition 2, the maximum arrangement corresponding to $\left(Y \cup\left\{a_{1}, a_{2 n-1}\right\}, X \cup\left\{a_{2}, a_{2 n}\right\}\right)$ is in the following form:

\begin{tabular}{c|c|c|c|c|c}
\hline$\cdots$ & $a_{2 n-2}$ & $a_{1}$ & $a_{2 n-1}$ & $a_{4}$ & $\cdots$ \\
\hline$\cdots$ & $a_{3}$ & $a_{2 n}$ & $a_{2}$ & $a_{2 n-3}$ & $\cdots$ \\
\hline
\end{tabular}

Let $\Delta=\left|a_{1}-a_{2 n}\right|^{q}+\left|a_{1}-a_{2 n-1}\right|^{q}+\left|a_{1}-a_{2 n-2}\right|^{q}+\left|a_{2}-a_{2 n}\right|^{q}+\left|a_{2}-a_{2 n-1}\right|^{q}+\left|a_{2}-a_{2 n-3}\right|^{q}$ $+\left|a_{3}-a_{2 n}\right|^{q}+\left|a_{4}-a_{2 n-1}\right|^{q}-\left|a_{3}-a_{2 n-3}\right|^{q}-\left|a_{4}-a_{2 n-2}\right|^{q}$. It is easy to check that $r_{q}(Y \cup$ $\left.\left\{a_{1}, a_{2 n-1}\right\}, X \cup\left\{a_{2}, a_{2 n}\right\}\right)=\Delta+r_{q}(X, Y)$.

By way of contradiction. Assume $\left(Y \cup\left\{a_{1}, a_{2 n-1}\right\}, X \cup\left\{a_{2}, a_{2 n}\right\}\right)$ is not maximum. By proposition 3 , there exists a maximum arrangement in the following form:

\begin{tabular}{c|c|c|c|c|c}
\hline$\cdots$ & $a_{2 n-2}$ & $a_{1}$ & $a_{2 n-1}$ & $x_{2}$ & $\cdots$ \\
\hline$\cdots$ & $a_{3}$ & $a_{2 n}$ & $a_{2}$ & $y_{n-1}$ & $\cdots$ \\
\hline
\end{tabular}

Let $\left(a_{1} a_{2 n-2} \cdots x_{2} a_{2 n-1}, a_{2 n} a_{3} \cdots y_{n-1} a_{2}\right)$ be the arrangement above and $\Delta^{\prime}=\left|a_{1}-a_{2 n}\right|^{q}$ $+\left|a_{1}-a_{2 n-1}\right|^{q}+\left|a_{1}-a_{2 n-2}\right|^{q}+\left|a_{2}-a_{2 n}\right|^{q}+\left|a_{2}-a_{2 n-1}\right|^{q}+\left|a_{2}-y_{n-1}\right|^{q}+\left|a_{3}-a_{2 n}\right|^{q}+$ $\left|x_{2}-a_{2 n-1}\right|^{q}-\left|a_{3}-y_{n-1}\right|^{q}-\left|x_{2}-a_{2 n-2}\right|^{q}$. Again it is clear that $r_{q}\left(a_{1} a_{2 n-2} \cdots x_{2} a_{2 n-1}, a_{2 n}\right.$ $\left.a_{3} \cdots y_{n-1} a_{2}\right)=\Delta^{\prime}+r_{q}\left(a_{2 n-2} \cdots x_{2}, a_{3} \cdots y_{n-1}\right)$. Since $(X, Y)$ is maximum for the subproblem $\left\{a_{3}, \cdots, a_{2 n-2}\right\}, r_{q}(X, Y) \geq r_{q}\left(a_{2 n-2} \cdots x_{2}, a_{3} \cdots y_{n-1}\right)$. It implies $\Delta<\Delta^{\prime}$. But

$$
\begin{aligned}
& \Delta-\Delta^{\prime} \\
= & \left|a_{2}-a_{2 n-3}\right|^{q}+\left|a_{4}-a_{2 n-1}\right|^{q}-\left|a_{3}-a_{2 n-3}\right|^{q}-\left|a_{4}-a_{2 n-2}\right|^{q} \\
& -\left|a_{2}-y_{n-1}\right|^{q}-\left|x_{2}-a_{2 n-1}\right|^{q}+\left|a_{3}-y_{n-1}\right|^{q}+\left|x_{2}-a_{2 n-2}\right|^{q} \\
\geq & \left|a_{4}-a_{2 n-1}\right|^{q}-\left|a_{4}-a_{2 n-2}\right|^{q}-\left|x_{2}-a_{2 n-1}\right|^{q}+\left|x_{2}-a_{2 n-2}\right|^{q} \\
\geq & 0
\end{aligned}
$$

where the first inequality holds because $a_{2} \leq a_{3}$ and $y_{n-1} \leq a_{2 n-3}$ and the second holds because $a_{4} \leq x_{2}$ and $a_{2 n-2} \leq a_{2 n-1}$. A contradiction!

With the "moreover" part proved, the rest is to prove $\left(\left\{a_{1}, a_{4}, a_{6}, a_{7}\right\},\left\{a_{2}, a_{3}, a_{5}, a_{8}\right\}\right)$ is maximum. Suppose not, then by proposition 3 and theorem 2 , the only possible maximum arrangement is $\left(a_{1} a_{6} a_{5} a_{7}, a_{8} a_{3} a_{4} a_{2}\right)$. But $r_{q}\left(a_{1} a_{6} a_{5} a_{7}, a_{8} a_{3} a_{4} a_{2}\right)-r_{q}\left(a_{1} a_{6} a_{4} a_{7}, a_{8} a_{3} a_{5} a_{2}\right)=$ $\left|a_{6}-a_{5}\right|^{q}+\left|a_{7}-a_{5}\right|^{q}-\left|a_{6}-a_{4}\right|^{q}-\left|a_{7}-a_{4}\right|^{q}+\left|a_{2}-a_{4}\right|^{q}+\left|a_{3}-a_{4}\right|^{q}-\left|a_{2}-a_{5}\right|^{q}-$ $\left|a_{3}-a_{5}\right|^{q} \leq 0$ due to $a_{4} \leq a_{5}$. A contradiction! Thus the proposition holds for $n=4$ as well.

\section{Conclusion}

We have resolved the 2-dartboard problem. However, it is still not clear how to solve the $k$-dartboard problem when $k>2$. It will be interesting to design an efficient algorithm for it or prove it to be hard, say NP-hard, etc. 


\section{Acknowledgments}

The authors would like to thank the anonymous referees for their helpful comments. The work was supported in part by the National Science Council of Taiwan under contract NSC 95-2221-E-009-034.

\section{References}

[1] G. Carpaneto, P. Toth, Some new branching and bounding criteria for the asymmetric traveling salesman problem, Management Science, 26: 736-743, 1980.

[2] Chern-Ching Chao, Wen-Qi Liang, Arranging $n$ distinct numbers on a line or a circle to reach extreme total variations, European J. Combin., 13: 325-334, 1992.

[3] G.L. Cohen, E. Tonkes, Dartboard arrangements, Electron J. Combin., 8(2): R4, 2001.

[4] S.A. Curtis, Darts and hoopla board design, Information Processing Letters, 92: 53-56, 2004.

[5] H.A. Eiselt, G. Laporte, A combinatorial optimization problem arising in dartboard design, J. Oper. Res. Soc., 42(2): 113-118, 1991.

[6] C. Papadimitriou and K. Steiglitz Combinatorial Optimization, algorithms and complexity, Prentice-Hall Inc., 1982.

[7] K. Selkirk, Re-designing the dartboard, The Mathematical Gazette, 60(413): 171178, 1976.

[8] D. Singmaster, Arranging a dartboard, Bulletin of the Institute of Mathematics and its Applications, 16: 93-97, 1980. 